\title{
The Cascadic Multigrid Method of the Weak Galerkin Method for Second-Order Elliptic Equation
}

\author{
Shi Sun, ${ }^{1}$ Ziping Huang, ${ }^{1}$ Cheng Wang, ${ }^{1}$ and Liming Guo ${ }^{2}$ \\ ${ }^{1}$ School of Mathematical Sciences, Tongji University, Shanghai 200092, China \\ ${ }^{2}$ School of Mathematical and Statistics, Xinyang Normal University, Xinyang 464000, China \\ Correspondence should be addressed to Cheng Wang; wangcheng@tongji.edu.cn
}

Received 28 April 2017; Accepted 17 September 2017; Published 18 October 2017

Academic Editor: Petr Krysl

Copyright (c) 2017 Shi Sun et al. This is an open access article distributed under the Creative Commons Attribution License, which permits unrestricted use, distribution, and reproduction in any medium, provided the original work is properly cited.

\begin{abstract}
This paper is devoted to the analysis of the cascadic multigrid algorithm for solving the linear system arising from the weak Galerkin finite element method. The proposed cascadic multigrid method is optimal for conjugate gradient iteration and quasi-optimal for Jacobi, Gauss-Seidel, and Richardson iterations. Numerical results are also provided to validate our theoretical analysis.
\end{abstract}

\section{Introduction}

The weak Galerkin (WG) finite element method (FEM) is a recently developed numerical method for solving various types of partial differential equations. A new concept of the discrete weak gradient is introduced, which is the most significant feature of the weak Galerkin method. Due to the definition of weak gradient, the weak Galerkin finite element method is flexible in numerical approximation.

There have been some studies and applications of the weak Galerkin finite element method. The method was first introduced by Wang and Ye in [1] for second-order elliptic problems. The corresponding numerical analysis of the weak Galerkin method based on Raviart-Thomas (RT) elements and Brezzi-Douglas-Marini (BDM) elements is given in [2]. A stabilization technique was presented and applied to the weak Galerkin finite element method, and the resulting weak Galerkin finite element method is no longer limited to RT and BDM elements [3]. In [4], the weak Galerkin mixed finite element method for biharmonic equations has been developed. For the applications of the weak Galerkin finite element method for other types of partial differential equations, the readers are referred to [5-8].

In this paper, we consider the cascadic multigrid method for solving the linear system generated by the weak Galerkin finite element method for second-order elliptic problems. Multigrid methods [9] have been shown to be very effective in solving large scale system theoretically and numerically. The cascadic multigrid method $[10,11]$ is a one-way multigrid method and easy to be implemented since it requires no coarse grid corrections at all. Much effort has been made to the analysis of cascadic multigrid method (see, e.g., $[12,13]$ ). Following the idea of [13], we can establish the error estimate in energy norm and the computational complexity estimate of the proposed cascadic multigrid method.

The rest of this paper is organized as follows. In Section 2, we introduce the weak Galerkin finite element method for second-order elliptic problems. In Section 3, a cascadic multigrid algorithm based on the weak Galerkin finite element discretization is proposed and analyzed, and the error estimates in energy norm and computational complexity are obtained. Numerical experiments are conducted to confirm our theoretical results in Section 4. Finally, we give the conclusion in Section 5.

\section{Model Problem and Its WG Finite Element Approximation}

Consider the following second-order elliptic problem:

$$
\begin{array}{rlrl}
-\nabla \cdot(\mathbb{A}(x) \nabla u) & =f & & \text { in } \Omega, \\
u=0 & & \text { on } \partial \Omega,
\end{array}
$$


where $\Omega$ is a convex polygonal domain with boundary $\partial \Omega$ in $\mathbb{R}^{2}, \mathbb{A} \in\left[L^{\infty}(\Omega)\right]^{2 \times 2}, f \in L^{2}(\Omega)$. Furthermore, assume that $\mathbb{A}$ is symmetric uniformly positive definite and uniformly bounded-above diffusion; namely, there exist positive constants $\alpha$ and $\beta$ such that

$$
\alpha \xi^{T} \xi \leq \xi^{T} \mathbb{A}(x) \xi \leq \beta \xi^{T} \xi, \quad \forall \xi \in \mathbb{R}^{2}, x \in \Omega .
$$

Here and thereafter, for any subset $D \subseteq \mathbb{R}^{2}$, we use the standard notations for the Sobolev spaces $H^{s}(D)$ and $H_{0}^{s}(D)$ with $s \geq 0$. The inner-product, norm, and seminorm in $H^{s}(D)$ are denoted by $(\cdot, \cdot)_{s, D},\|\cdot\|_{s, D}$ and $|\cdot|_{s, D}$, respectively, and we skip the subscript $D$ when $D=\Omega$.

Since the domain $\Omega$ is convex, the unique solution $u$ of problem (1) exists and satisfies the full regularity assumption [14]

$$
\|u\|_{2} \leq C\|f\|_{0} .
$$

Let $K$ be a polygonal domain with interior $K^{0}$ and boundary $\partial K$. Denote by $W(K)$ the space of weak functions on $K$; that is,

$W(K)$

$$
:=\left\{v=\left\{v_{0}, v_{b}\right\}: v_{0} \in L^{2}(K), v_{b} \in H^{1 / 2}(\partial K)\right\} .
$$

For any $v \in W(K)$, the weak gradient of $v$ is defined as $\nabla_{w} v$,

$$
\begin{aligned}
\left(\nabla_{w} v, \mathbf{q}\right)_{K}=-\left(v_{0}, \nabla \cdot \mathbf{q}\right)_{K}+\left\langle v_{b}, \mathbf{q} \cdot \mathbf{n}\right\rangle_{\partial K}, & \\
& \forall \mathbf{q} \in H(\operatorname{div}, K),
\end{aligned}
$$

where $H(\operatorname{div}, K)=\left\{\mathbf{q}: \mathbf{q} \in\left[L^{2}(K)\right]^{2}, \nabla \cdot \mathbf{q} \in L^{2}(K)\right\}$.

The discrete weak gradient, denoted by $\nabla_{w, r, K} v \in$ $\left[P_{r}(K)\right]^{2}$, is defined as follows:

$$
\begin{aligned}
&\left(\nabla_{w, r, K} v, \mathbf{q}\right)_{K}=-\left(v_{0}, \nabla \cdot \mathbf{q}\right)_{K}+\left\langle v_{b}, \mathbf{q} \cdot \mathbf{n}\right\rangle_{\partial K}, \\
& \forall \mathbf{q} \in\left[P_{r}(K)\right]^{2} .
\end{aligned}
$$

Let $T_{h}$ be a shape-regular, quasi-uniform triangular mesh of the domain $\Omega$, with the mesh size $h$. $C$ denotes a generic positive constant independent of the mesh size $h$ throughout this paper. Denote the weak function space on $T_{h}$ by $V$; that is,

$$
V:=\left\{v=\left\{v_{0}, v_{b}\right\}:\left.\left\{v_{0}, v_{b}\right\}\right|_{T} \in W(T), \forall T \in T_{h}\right\} .
$$

For any given integer $k \geq 1$, define $W_{k}(T)$ as the discrete weak function space consisting of polynomials of degree $k$ in $T$ and piecewise polynomials of degree $k$ on $\partial T$; that is,

$$
\begin{aligned}
& W_{k}(T):=\left\{v=\left\{v_{0}, v_{b}\right\}:\left.v_{0}\right|_{T} \in P_{k}(T),\left.v_{b}\right|_{e}\right. \\
& \left.\quad \in P_{k}(e), e \in \partial T\right\} .
\end{aligned}
$$

The weak Galerkin finite element spaces are defined as follows:

$$
\begin{aligned}
V_{h} & :=\left\{v=\left\{v_{0}, v_{b}\right\}:\left.\left\{v_{0}, v_{b}\right\}\right|_{T} \in W_{k}(T), \forall T \in T_{h}\right\}, \\
V_{h}^{0} & :=\left\{v: v \in V_{h}, v_{b}=0 \text { on } \partial \Omega\right\} .
\end{aligned}
$$

It follows from [3] that

$$
\left.\nabla_{w, k-1} v\right|_{T}=\nabla_{w, k-1}\left(\left.v\right|_{T}\right), \quad \forall v \in V_{h}, T \in T_{h} .
$$

For the discrete weak gradient, we will drop the subscript $k-1$ in the notation $\nabla_{w, k-1}$ for simplicity.

The weak Galerkin finite element method can be written as to find $u_{h}=\left\{u_{0}, u_{b}\right\} \in V_{h}^{0}$ such that

$$
a_{h}\left(u_{h}, v\right)=\left(f, v_{0}\right), \quad \forall v=\left\{v_{0}, v_{b}\right\} \in V_{h}^{0},
$$

where

$$
\begin{aligned}
a_{h}(w, v)= & \sum_{T \in T_{h}}\left(\mathbb{A}(x) \nabla_{w} w, \nabla_{w} v\right)_{T} \\
& +h_{T}^{-1}\left\langle w_{0}-w_{b}, v_{0}-v_{b}\right\rangle_{\partial T},
\end{aligned}
$$

for any $w=\left\{w_{0}, w_{b}\right\}, v=\left\{v_{0}, v_{b}\right\} \in V$.

For each element $T \in T_{h}$, denote by $Q_{0}$ the $L^{2}$ projection from $L^{2}(T)$ onto $P_{k}(T) . E_{h}$ is the set of all edges in $T_{h}$. For each edge $e \in E_{h}$, let $Q_{b}$ be the $L^{2}$ projection from $L^{2}(e)$ onto $P_{k}(e)$. Define $Q_{h}: V \rightarrow V_{h}$

$$
Q_{h} v:=\left\{Q_{0} v_{0}, Q_{b} v_{b}\right\}, \quad \forall v=\left\{v_{0}, v_{b}\right\} \in V .
$$

Denote by $\mathbb{Q}_{h}$ the $L^{2}$ projection onto the local discrete gradient space $\left[P_{k-1}(T)\right]^{2}$. Lemma 5.1 in [3] shows that, on each $T \in T_{h}$,

$$
\nabla_{w}\left(Q_{h} \phi\right)=\mathbb{Q}_{h}(\nabla \phi), \quad \forall \phi \in H^{1}(\Omega) .
$$

For $Q_{h}$ and $\mathbb{Q}_{h}$ defined above, the following lemmas provide some estimates.

Lemma 1 (see [3], Lemma 5.2). For any $\phi \in H^{k+1}(\Omega)$, we have

$$
\begin{aligned}
& \sum_{T \in T_{h}}\left\|\phi-Q_{0} \phi\right\|_{0, T}^{2}+\sum_{T \in T_{h}} h_{T}^{2}\left\|\nabla\left(\phi-Q_{0} \phi\right)\right\|_{0, T}^{2} \\
& \leq C h^{2(k+1)}\|\phi\|_{k+1}^{2}, \\
& \sum_{T \in T_{h}}\left\|\mathbb{A}\left(\nabla \phi-\mathbb{Q}_{h}(\nabla \phi)\right)\right\|_{0, T}^{2} \leq C h^{2 k}\|\phi\|_{k+1}^{2} .
\end{aligned}
$$

Lemma 2 (see [3], Lemma 5.3). For any $w \in H^{k+1}(\Omega)$ and $v=\left\{v_{0}, v_{b}\right\} \in V_{h}$, we have

$$
\begin{aligned}
& \left|\sum_{T \in T_{h}} h_{T}^{-1}\left\langle Q_{0} w-Q_{b} w, v_{0}-v_{b}\right\rangle_{\partial T}\right| \\
& \quad \leq C h^{k}\|w\|_{k+1} \mid\|v\| \|, \\
& \left|\sum_{T \in T_{h}}\left\langle\mathbb{A}\left(\nabla w-\mathbb{Q}_{h} \nabla w\right) \cdot \mathbf{n}, v_{0}-v_{b}\right\rangle_{\partial T}\right| \\
& \quad \leq C h^{k}\|w\|_{k+1}|\|v\||,
\end{aligned}
$$

where $\|\cdot\| \cdot \|$ is the energy norm; that is, for any $v \in V_{h},\|\| v\|\|^{2}=$ $a_{h}(v, v)$. 
Lemma 3 (see [3], Theorem 8.1, Theorem 8.2). Assume the exact solution $u \in H^{k+1}(\Omega)$; then we have

$$
\begin{gathered}
\left\|u_{h}-Q_{h} u\right\| \leq C h^{k}\|u\|_{k+1}, \\
\left\|Q_{0} u-u_{0}\right\|_{0} \leq C h^{k+1}\|u\|_{k+1} .
\end{gathered}
$$

For any $v=\left\{v_{0}, v_{b}\right\}, w=\left\{w_{0}, w_{b}\right\} \in V$, we define an inner-product by

$$
\begin{aligned}
((v, w))= & \sum_{T \in T_{h}}\left(v_{0}, w_{0}\right)_{T} \\
& +\sum_{T \in T_{h}} h_{T}\left\langle v_{0}-v_{b}, w_{0}-w_{b}\right\rangle_{\partial T} .
\end{aligned}
$$

Define the following discrete norms:

$$
\begin{aligned}
\|v\|_{0, h, T}^{2} & =\left\|v_{0}\right\|_{0, T}^{2}+h_{T}\left\|v_{0}-v_{b}\right\|_{0, \partial K}^{2}, \quad \forall T \in T_{h}, \\
\|v\|_{0, h}^{2} & =\left(\sum_{T \in T_{h}}\|v\|_{0, h, T}^{2}\right)^{1 / 2} .
\end{aligned}
$$

It is clear that $\|v\|_{0, h}^{2}=((v, v))$.

With the above estimates, the following lemma can be proved, which is needed in Section 3.

Lemma 4. Let $u \in H^{k+1}(\Omega)$ be the exact solution of problem (1), and let $u_{h}=\left\{u_{0}, u_{b}\right\}$ be the weak Galerkin finite element solution of problem (11); then we have

$$
\begin{gathered}
\left\|u-u_{h}\right\|\left\|\leq C h^{k}\right\| u \|_{k+1}, \\
\left\|u-u_{h}\right\|_{0, h} \leq C h^{k+1}\|u\|_{k+1} .
\end{gathered}
$$

Proof. Apparently, for any edge $e \in E_{h}$, we have

$$
\left\|Q_{0} u-Q_{b} u\right\|_{e} \leq\left\|Q_{0} u-u\right\|_{e} .
$$

$T$ is an element with $e$ as an edge. For any function $\varphi \in H^{1}(T)$, the following trace inequality is well known

$$
\|\varphi\|_{e}^{2} \leq C\left(h_{T}^{-1}\|\varphi\|_{0, T}^{2}+h_{T}\|\nabla \varphi\|_{0, T}^{2}\right) .
$$

Using the trace inequalities (26) and (15), we have

$$
\begin{aligned}
& \sum_{T \in T_{h}}\left\|Q_{0} u-Q_{b} u\right\|_{0, \partial T}^{2} \leq\left\|Q_{0} u-u\right\|_{0, \partial T}^{2} \\
& \quad \leq \sum_{T \in T_{h}} C h_{0, T}^{-1}\left(\left\|Q_{0} u-u\right\|_{T}^{2}+h_{T}^{2}\left\|\nabla\left(Q_{0} u-u\right)\right\|_{0, T}^{2}\right) \\
& \quad \leq C h^{2 k+1}\|u\|_{k+1}^{2} .
\end{aligned}
$$

Then it follows from (14) and (16) that

$$
\begin{aligned}
& \sum_{T \in T_{h}}\left(\mathbb{A}\left(\nabla u-\nabla_{w}\left(Q_{h} u\right)\right), \nabla u-\nabla_{w}\left(Q_{h} u\right)\right) \\
& \quad \leq C h^{2 k}\|u\|_{k+1}^{2} .
\end{aligned}
$$

Thus, we have

$$
\begin{aligned}
\| u- & Q_{h} u\|\|^{2} \\
= & \sum_{T \in T_{h}}\left(\mathbb{A}\left(\nabla u-\nabla_{w}\left(Q_{h} u\right)\right), \nabla u-\nabla_{w}\left(Q_{h} u\right)\right)_{T} \\
& \quad+h_{T}^{-1}\left\|Q_{0} u-Q_{b} u\right\|_{0, \partial T}^{2} \leq C h^{2 k}\|u\|_{k+1}^{2} .
\end{aligned}
$$

By using the triangle inequality, we get from (19) that

$$
\begin{aligned}
\left\|u_{h}-u\right\| & \leq\left\|\mid u_{h}-Q_{h} u\right\|+\|\| Q_{h} u-u\|\| \\
& \leq C h^{k}\|u\|_{k+1} .
\end{aligned}
$$

According to the definition of $\|\cdot\|_{0, h}$ and $\||\cdot|\|$, we have

$$
\begin{aligned}
\left\|u-u_{h}\right\|_{0, h}^{2}= & \left\|u-u_{0}\right\|_{0}^{2} \\
& +\sum_{T \in T_{h}} h_{T}^{2} h_{T}^{-1}\left\|\left(u-u_{h}\right)_{0}-\left(u-u_{h}\right)_{b}\right\|_{0, \partial T}^{2} \\
\leq & \left\|u-u_{0}\right\|_{0}^{2}+h^{2}\left\|u-u_{h}\right\|^{2} .
\end{aligned}
$$

It follows from (15) and (20) that

$$
\begin{aligned}
\left\|u-u_{0}\right\|_{0} & \leq\left\|u-Q_{0} u\right\|_{0}+\left\|Q_{0} u-u_{0}\right\|_{0} \\
& \leq C h^{k+1}\|u\|_{k+1} .
\end{aligned}
$$

Combining the above three inequalities, we obtain the aimed result (24).

\section{Cascadic Multigrid Algorithm}

In this section, the error estimate and computational complexity of the cascadic multigrid method are analyzed.

Assume that $T_{h_{l}}(l \geq 0)$ is a triangular partition of $\Omega$ with the mesh size $h_{l}, E_{h_{l}}$ is the set of all edges in $T_{h_{l}}$, and $V_{h_{l}}$ is the corresponding weak discrete space on mesh $T_{h_{l}}$. Noting that $T_{l+1}$ is obtained by connecting the midpoints of three edges of all triangles in $T_{l}$, we have $h_{l}=2^{-l} h_{0}$, where $h_{0}$ is the mesh size of $T_{0}$. For simplicity, define $T_{l}:=T_{h_{l}}, E_{l}:=E_{h_{l}}, V_{l}:=V_{h_{l}}^{0}$, $\|\cdot\|_{0, h_{l}}:=\|\cdot\|_{0, l}$, and $\left\|\left|\cdot\|\|_{h_{l}}:=\||\cdot|\|_{l}\right.\right.$. The weak Galerkin finite element approximation of problem (11) on level $l$ can be rewritten as to find $u_{l}=\left\{u_{0}, u_{b}\right\} \in V_{l}$ such that

$$
\begin{aligned}
& a_{l}\left(u_{l}, v_{l}\right):=a_{h_{l}}\left(u_{l}, v_{l}\right)=\left(f, v_{0}\right), \\
& \forall v_{l}=\left\{v_{0}, v_{b}\right\} \in V_{l} .
\end{aligned}
$$

Define an intergrid transfer operator $I_{l}: V_{l-1} \rightarrow V_{l}$, for any $v \in V_{l-1}$

(1) If $x \in K_{l}^{0}$ and the element $K_{l} \in T_{l}$ is obtained by refining $K_{l-1} \in T_{l-1}$, then

$$
\left(I_{l} v\right)_{0}(x)=v_{0}(x) \text {. }
$$

(2) If $x \in e_{l}$ and edge $e_{l} \in E_{l}$ locates in the interior of $K_{l-1} \in T_{l-1}$, then

$$
\left(I_{l} v\right)_{b}(x)=v_{0}(x) .
$$


(3) If $x \in e_{l}$ and edge $e_{l} \in E_{l}$ is part of edge $e_{l-1} \in E_{l-1}$, then

$$
\left(I_{l} v\right)_{b}(x)=v_{b}(x) .
$$

Then the cascadic multigrid method can be written as follows.

Step 1. Set $u_{0}^{0}=u_{0}^{*}=u_{0}$.

Step 2. For $l=1, \ldots, L$, set $u_{l}^{0}=I_{l} u_{l-1}^{*}$, and do iterations

$$
u_{l}^{m_{l}}=C_{l}^{m_{l}} u_{l}^{0} \text {. }
$$

Step 3. Set $u_{l}^{*}=u_{l}^{m_{l}}$.

The notation $C_{l}$ in Step 2 denotes the iterative operator on level $l$. For the operator $C_{l}$, we assume that there exists a linear operator $R_{l}: V_{l} \rightarrow V_{l}$ such that

$$
\begin{aligned}
& u_{l}-C_{l}^{m_{l}} u_{l}^{0}=R_{l}^{m_{l}}\left(u_{l}-u_{l}^{0}\right), \\
& \left\|R_{l}^{m_{l}} v\right\|_{l} \leq C \frac{h_{l}^{-1}}{m_{l}^{\gamma}}\|v\|_{0, l}, \quad \forall v \in V_{l}, \\
& \left\|R_{l}^{m_{l}} v\right\|_{l} \leq\|\mid v\|_{l}, \quad \forall v \in V_{l},
\end{aligned}
$$

where $m_{l}$ represents the number of iteration steps on level $l$ and the parameter $0 \leq \gamma \leq 1$. As a matter of fact, the assumptions above hold for the Richardson, Jacobi, and Gauss-Seidel iterations with $\gamma=1 / 2$ and for conjugate gradient iteration with $\gamma=1$. We refer to $[9,13]$ for details on these results.

The following two lemmas can be proved based on the definition of $I_{l}$.

Lemma 5. $I_{l}$ is the intergrid transfer operator. For any $v=$ $\left\{v_{0}, v_{b}\right\} \in V_{l-1}$, we have

$$
\begin{aligned}
\left\|I_{l} v\right\|_{0, l} & \leq\|v\|_{0, l-1}, \\
\left\|I_{l} v\right\|_{l} & \leq C\|\| v\|\|_{l-1} .
\end{aligned}
$$

Proof. For any element $K_{l-1} \in T_{l-1}$, let $E_{l}^{0}$ be the collection of edges $e_{l}$ located in $K_{l-1}$. It follows from the definition of $I_{l}$ that

$$
\left\|\left(I_{l} v\right)_{0}-\left(I_{l} v\right)_{b}\right\|_{0, e_{l}}=0, \quad \forall e_{l} \in E_{l}^{0} .
$$

Thus, we have

$$
\begin{aligned}
& \left\|I_{l} v\right\|_{0, l}^{2}=\sum_{K_{l} \in T_{l}}\left(\left\|\left(I_{l} v\right)_{0}\right\|_{0, K_{l}}^{2}\right. \\
& \left.+\sum_{e_{l} \in \partial K_{l}} h_{l}\left\|\left(I_{l} v\right)_{0}-\left(I_{l} v\right)_{b}\right\|_{0, e_{l}}^{2}\right) \\
& =\sum_{K_{l-1} \in T_{l-1}}\left(\left\|\left(I_{l} v\right)_{0}\right\|_{0, K_{l-1}}^{2}\right. \\
& \left.+\sum_{e_{l-1} \in \partial K_{l-1}} h_{l}\left\|\left(I_{l} v\right)_{0}-\left(I_{l} v\right)_{b}\right\|_{0, e_{l-1}}^{2}\right)
\end{aligned}
$$

$$
\begin{aligned}
& \leq \sum_{K_{l-1} \in T_{l-1}}\left\|\left(I_{l} v\right)_{0}\right\|_{0, K_{l-1}}^{2}+\sum_{K_{l-1} \in T_{l-1}} h_{l-1} \|\left(I_{l} v\right)_{0} \\
& -\left(I_{l} v\right)_{b}\left\|_{0, \partial K_{l-1}}^{2}=\right\| v \|_{0, l-1}^{2} .
\end{aligned}
$$

For any $K \in T_{l-1}$, from the definition of weak gradient (5), we have

$$
\begin{aligned}
\left(\nabla v_{0}, \mathbf{q}\right)_{K}=\left(\nabla_{w} v, \mathbf{q}\right)_{K}+\left\langle v_{0}-v_{b}, \mathbf{q} \cdot \mathbf{n}\right\rangle_{\partial K}, & \\
& \forall \mathbf{q} \in H(\operatorname{div}, \Omega) .
\end{aligned}
$$

If $\varphi$ is a polynomial in $K$, from trace inequality (26) and the standard inverse inequality, we have

$$
\|\varphi\|_{e}^{2} \leq C h_{K}^{-1}\|\varphi\|_{0, K}^{2} .
$$

Setting $\mathbf{q}=\nabla v_{0}$, with (44), we have

$$
\begin{aligned}
\left\|\nabla v_{0}\right\|_{K}^{2}= & \left(\nabla_{w} v, \nabla v_{0}\right)_{K}+\left\langle v_{0}-v_{b}, \nabla v_{0} \cdot \mathbf{n}\right\rangle_{\partial K} \\
\leq & \left\|\nabla_{w} v\right\|_{0, K}\left\|\nabla v_{0}\right\|_{0, K} \\
& +\left(h_{K}^{-1 / 2}\left\|v_{0}-v_{b}\right\|_{0, \partial K}\right)\left(h_{K}^{1 / 2}\left\|\nabla v_{0} \cdot \mathbf{n}\right\|_{0, \partial K}\right) \\
\leq & C\|v\|\left\|_{K}\right\| \nabla v_{0} \|_{0, K} .
\end{aligned}
$$

This implies

$$
\left\|\nabla v_{0}\right\|_{0, K} \leq C\||| v \mid\|_{K} .
$$

Note that $K$ consisted of some elements in $T_{l}$; that is, there exist $K_{i} \in T_{l}, i=1, \ldots, m$, such that $K=\cup_{i=1}^{m} K_{i}$.

Application of (44) yields

$$
\left\|\nabla_{w}\left(I_{l} v\right) \cdot \mathbf{n}\right\|_{0, \partial K_{i}} \leq C h_{K_{i}}^{-1 / 2}\left\|\nabla_{w}\left(I_{l} v\right)\right\|_{0, K_{i}},
$$

$\forall v \in V_{l-1}$.

By the definition of $\nabla_{w} v,(46)$ and (47), we have

$$
\begin{aligned}
&\left\|\nabla_{w}\left(I_{l} v\right)\right\|_{0, K_{i}}^{2}=\left(\nabla_{w}\left(I_{l} v\right), \nabla_{w}\left(I_{l} v\right)\right)_{K_{i}} \\
&=\left(\nabla\left(I_{l} v\right)_{0}, \nabla_{w}\left(I_{l} v\right)\right)_{K_{i}} \\
&-\left\langle\left(I_{l} v\right)_{0}-\left(I_{l} v\right)_{b}, \nabla_{w}\left(I_{l} v\right) \cdot \mathbf{n}\right\rangle_{\partial K_{i}} \\
& \leq\left\|\nabla v_{0}\right\|_{0, K_{i}}\left\|\nabla_{w}\left(I_{l} v\right)\right\|_{0, K_{i}} \\
&+\left\|\left(I_{l} v\right)_{0}-\left(I_{l} v\right)_{b}\right\|_{0, \partial K_{i}}\left\|\nabla_{w}\left(I_{l} v\right) \cdot \mathbf{n}\right\|_{0, \partial K_{i}} \\
& \leq\left\|\nabla v_{0}\right\|_{0, K}\left\|\nabla_{w}\left(I_{l} v\right)\right\|_{0, K_{i}} \\
&+\left\|v_{0}-v_{b}\right\|_{0, \partial K}\left\|\nabla_{w}\left(I_{l} v\right) \cdot \mathbf{n}\right\|_{0, \partial K_{i}} \\
& \leq\|v \mid\|\left\|_{K}\right\| \nabla_{w}\left(I_{l} v\right) \|_{0, K_{i}} \\
&+h_{K}^{1 / 2}\|\| v\|\|_{K} h_{K_{i}}^{-1 / 2}\left\|\nabla_{w}\left(I_{l} v\right)\right\|_{0, K_{i}} \\
& \leq C\|v\|\left\|_{K}\right\| \nabla_{w}\left(I_{l} v\right) \|_{0, K_{i}} \cdot
\end{aligned}
$$


Since

$$
\begin{gathered}
h_{K_{i}}^{-1}\left\|\left(I_{l} v\right)_{0}-\left(I_{l} v\right)_{b}\right\|_{0, \partial K_{i}}^{2} \leq h_{K_{i}}^{-1}\left\|v_{0}-v_{b}\right\|_{0, \partial K}^{2} \\
\leq C h_{K}^{-1}\left\|v_{0}-v_{b}\right\|_{0, \partial K}^{2} \leq C\|\| v \|\left.\right|_{K},
\end{gathered}
$$

we obtain

$$
\begin{aligned}
& \|\| I_{l} v \|_{l}^{2} \\
& =\sum_{T \in T_{l}}\left(A \nabla_{w}\left(I_{l} v\right), \nabla_{w}\left(I_{l} v\right)\right)_{T} \\
& \quad+h_{T}^{-1}\left\langle\left(I_{l} v\right)_{0}-\left(I_{l} v\right)_{b},\left(I_{l} v\right)_{0}-\left(I_{l} v\right)_{b}\right\rangle_{\partial T} \\
& \leq C \sum_{T \in T_{l}}\left(\left\|\nabla_{w}\left(I_{l} v\right)\right\|_{0, T}^{2}+h_{T}^{-1}\left\|\left(I_{l} v\right)_{0}-\left(I_{l} v\right)_{b}\right\|_{0, \partial T}^{2}\right) \\
& \leq C\|\| v\|\|_{l-1}^{2},
\end{aligned}
$$

which completes the proof of this lemma.

Lemma 6. Assume that $u_{l}$ and $u_{l-1}$ are the weak Galerkin finite element solutions associated with $V_{l}$ and $V_{l-1}$, respectively; then

$$
\left\|u_{l}-I_{l} u_{l-1}\right\|_{0, l} \leq C h_{l}^{2}\|f\|_{0} .
$$

Proof. Let $u_{l}^{F}$ and $u_{l-1}^{F}$ be the finite element solutions associated with $T_{l}$ and $T_{l-1}$, respectively. By the triangle inequality, (39), (24), and the regularity (3), we have

$$
\begin{aligned}
\left\|u_{l}-I_{l} u_{l-1}\right\|_{0, l} \leq & \left\|u_{l}-u\right\|_{0, l}+\left\|u-u_{l-1}^{F}\right\|_{0, l} \\
& +\left\|I_{l} u_{l-1}^{F}-I_{l} u_{l-1}\right\|_{0, l} \\
\leq & \left\|u_{l}-u\right\|_{0, l}+\left\|u-u_{l-1}^{F}\right\|_{0} \\
& +\left\|u_{l-1}^{F}-u_{l-1}\right\|_{0, l-1} \leq C h_{l}^{2}\|u\|_{2} \\
\leq & C h_{l}^{2}\|f\|_{0} .
\end{aligned}
$$

which completes the proof of this lemma.

Let $V_{l-1}^{F}$ be the standard finite element space associated with $T_{l-1}$. Define the projection operator $P_{l-1}: V_{l-1} \rightarrow V_{l-1}^{F}$ by

$$
a_{l}\left(P_{l-1} u, v\right)=a_{l}\left(I_{l} u, v\right), \quad \forall v \in V_{l-1}^{F} .
$$

It is easy to check that

$$
\begin{gathered}
a_{l}\left(P_{l-1} u, v\right)=a_{l-1}\left(P_{l-1} u, v\right), \quad v \in V_{l-1}^{F}, \\
a_{l}\left(I_{l} u, v\right)=a_{l-1}(u, v), \quad v \in V_{l-1}^{F}, \\
\left\|P_{l-1} v\right\|_{l} \leq\|\| v\|\|_{l-1}, \quad \forall v \in V_{l-1} .
\end{gathered}
$$

The following lemma is needed in the convergence analysis.

Lemma 7. For the projection operator $P_{l}$, we have

$$
\left\|I_{l} v-P_{l-1} v\right\|_{0, l} \leq C h_{l} \mid\|v\|_{l-1}, \quad \forall v \in V_{l-1} .
$$

Proof. Since $\Omega$ is a convex polygonal domain, for a given $v \epsilon$ $V_{l-1}$, we introduce an auxiliary problem, that is, to find $\xi \in$ $H^{2}(\Omega)$ such that

$$
\begin{aligned}
-\nabla \cdot(\mathbb{A} \nabla \xi) & =\left(I_{l} v-P_{l-1} v\right)_{0} \quad \text { in } \Omega, \\
\xi & =0 \quad \text { on } \partial \Omega .
\end{aligned}
$$

The solution $\xi$ satisfies the following inequality:

$$
\|\xi\|_{2} \leq\left\|\left(I_{l} v-P_{l-1} v\right)_{0}\right\|_{0}
$$

Let $\eta=\left\{\eta_{0}, \eta_{b}\right\}=I_{l} v-P_{l-1} v$. By the definition of $P_{l}$, we have

$$
a_{l}\left(I_{l} v-P_{l-1} v, w\right)=a_{l}(\eta, w)=\sum_{T \in T_{l}}\left(\nabla_{w} \eta, \nabla w\right)_{T},
$$

$$
\forall w \in V_{l-1}^{F}
$$

Then (58), the definition of weak gradient, and (14) give

$$
\begin{aligned}
\left\|\eta_{0}\right\|_{0}^{2}=-\sum_{T \in T_{l}}\left(\nabla \cdot(\mathbb{A} \nabla \xi), \eta_{0}\right)_{T} \\
=\sum_{T \in T_{l}}\left(\left(\mathbb{A} \mathbb{Q}_{l}(\nabla \xi), \nabla \eta_{0}\right)-\left\langle\mathbb{A} \nabla \xi \cdot \mathbf{n}, \eta_{0}\right\rangle_{\partial T}\right) \\
=\sum_{T \in T_{l}}\left(\left(-\nabla \cdot \mathbb{A} \mathbb{Q}_{l}(\nabla \xi), \eta_{0}\right)+\left\langle\mathbb{A} \mathbb{Q}_{l}(\nabla \xi) \cdot \mathbf{n}, \eta_{0}\right\rangle_{\partial T}\right. \\
\left.-\left\langle\mathbb{A} \nabla \xi \cdot \mathbf{n}, \eta_{0}\right\rangle_{\partial T}\right)=\sum_{T \in T_{l}}\left(\left(\mathbb{A} \nabla_{w}\left(Q_{l} \xi\right), \nabla_{w} \eta\right)\right. \\
+\left\langle\mathbb{A} \mathbb{Q}_{l}(\nabla \xi) \cdot \mathbf{n}-\mathbb{A} \nabla \xi \cdot \mathbf{n}, \eta_{0}-\eta_{b}\right\rangle_{\partial T} \\
\left.-\left\langle\mathbb{A} \nabla \xi \cdot \mathbf{n}, \eta_{b}\right\rangle_{\partial T}\right) .
\end{aligned}
$$

Since

$$
\sum_{T \in T_{l}}\left\langle A \nabla \xi \cdot \mathbf{n}, \eta_{b}\right\rangle_{\partial T}=0
$$

we get from (18) that

$$
\left\|\eta_{0}\right\|_{0}^{2} \leq a_{l}\left(Q_{l} \xi, \eta\right)+C h_{l}\|\xi\|_{2}\|\| \eta \|_{l} .
$$

For any $\xi_{l-1}^{F} \in V_{l-1}^{F}$, we have

$$
a_{l}\left(I_{l} v-P_{l-1} v, \xi_{l-1}^{F}\right)=0,
$$

which implies

$$
\begin{aligned}
\sum_{T \in T_{l}}\left(A \nabla_{w} \eta, \nabla \xi_{l-1}^{F}\right) & =\sum_{T \in T_{l}}\left(A \nabla_{w}\left(I_{l} v-P_{l-1} v\right), \nabla \xi_{l-1}^{F}\right) \\
& =0 .
\end{aligned}
$$


Thus, combining (16) and (18), we have

$$
\begin{aligned}
a_{l}\left(Q_{l} \xi, I_{l} v-P_{l-1} v\right)= & a_{l}\left(Q_{l} \xi-\xi_{l-1}^{F}, I_{l} v-P_{l-1} v\right) \\
\leq & \sum_{T \in T_{l}}\left(A \nabla_{w}\left(Q_{l} \xi-\xi\right), \nabla_{w} \eta\right)_{T} \\
& +\left(\mathbb{A} \nabla\left(\xi-\xi_{l-1}^{F}\right), \nabla_{w} \eta\right)_{T} \\
& +h_{T}^{-1}\left\langle Q_{0} \xi-Q_{b} \xi, \eta_{0}-\eta_{b}\right\rangle_{\partial T} \\
\leq & C h_{l}\|\xi\|_{2}\|\eta \eta\|_{l} .
\end{aligned}
$$

By using the regularity (60), we obtain

$$
\left\|\eta_{0}\right\|_{0}=\left\|\left(I_{l} v-P_{l-1} v\right)_{0}\right\|_{0} \leq C h_{l}\left\|I_{l} v-P_{l-1} v\right\| \|_{l} .
$$

Then it follows from (40) and (56) that

$$
\left\|\eta_{0}\right\|_{0} \leq C h_{l}\||v|\|_{l-1} .
$$

It is easy to show that

$$
\begin{aligned}
& \sum_{T \in T_{l}} h_{T}\left\langle\eta_{0}-\eta_{b}, \eta_{0}-\eta_{b}\right\rangle_{\partial T} \\
& \quad \leq h_{l}^{2} \sum_{T \in T_{l}} h_{T}^{-1}\left\langle\left(I_{l} v\right)_{0}-\left(I_{l} v\right)_{b},\left(I_{l} v\right)_{0}-\left(I_{l} v\right)_{b}\right\rangle_{\partial T} \\
& \quad \leq C h_{l}^{2} \sum_{T \in T_{l-1}} h_{T}^{-1}\left\langle v_{0}-v_{b}, v_{0}-v_{b}\right\rangle_{\partial T} \\
& \quad \leq C h_{l}^{2}\|\mid v\|_{l-1}^{2} .
\end{aligned}
$$

Combining (68) and (70), we obtain

$$
\begin{aligned}
\left\|I_{l} v-P_{l-1} v\right\|_{0, l}^{2} & =\left\|\eta_{0}\right\|_{0}^{2}+\sum_{T \in T_{l}} h_{T}\left\langle\eta_{0}-\eta_{b}, \eta_{0}-\eta_{b}\right\rangle_{\partial T} \\
& \leq C h_{l}^{2}\|\| v\|\|_{l-1}^{2},
\end{aligned}
$$

which completes the proof of this lemma.

The following two theorems are the main results of this paper, which can be proved in a similar way of [13] based on the above lemmas.

Theorem 8. If we take the CG iteration as the smoother, and the number of iterations on the level $l$ is the minimum integer satisfying

$$
m_{l} \geq \beta^{L-1} m_{L}
$$

with some fixed $\beta>1$ and $m_{L}$, then the cascadic multigrid method is optimal: that is, the error

$$
\left\|u_{L}-u_{L}^{*}\right\|_{L} \approx\left\|u-u_{L}\right\|_{L}
$$

and the computational complexity

$$
\text { amount of work }=O\left(n_{L}\right) \text {, }
$$

where $n_{l}$ denotes the dimension of the space $V_{L}$ on level $l$.
Theorem 9. If we take the Richardson, Jacobi, and GaussSeidel iterations as the smoother, the number of iterations on the level $l$ is the minimum integer satisfying

$$
m_{l} \geq \beta^{L-1} m_{L}
$$

while the number of iterations on the level $l$ is the minimum integer satisfying

$$
m_{L} \geq\left[m_{*} L^{2}\right]
$$

with some fixed $m_{*} \geq 1$, then the cascadic multigrid method is quasi-optimal: that is, the error

$$
\left\|\mid u_{L}-u_{L}^{*}\right\|_{L} \leq C \frac{h_{L}}{m_{*}^{1 / 2}}\|f\|
$$

and the computational complexity

$$
\sum_{k=1}^{L} m_{k} n_{k} \leq C m_{*} n_{L}\left(1+\log n_{L}\right)^{3} .
$$

\section{Numerical Examples}

In this section, we give some numerical experiments of the cascadic multigrid algorithm based on the weak Galerkin finite element method to verify the theoretical results proved in Section 3.

For simplicity, we choose $k=1$; that is, the weak Galerkin finite element space is

$$
\begin{aligned}
V_{l} & =\left\{v=\left\{v_{0}, v_{b}\right\}, v_{0} \in P_{1}(T), v_{b} \in P_{1}(\partial T), v_{b}\right. \\
& \left.=0 \text { on } \partial \Omega, \forall T \in T_{l}\right\},
\end{aligned}
$$

and the weak gradient space is

$$
G_{l}=\left\{\mathbf{q} \in\left[P_{0}(T)\right]^{2}, \forall T \in T_{l}\right\} .
$$

For the model problem (1) in the domain $\Omega=(0,1)^{2}$, we give the following numerical examples.

Case 1. Let the exact solution $u=\sin (\pi x) \sin (\pi y)$ and set $\mathbb{A}(x, y)=e^{x+y}$. The numerical results are reported in Table 1.

Case 2. Let the exact solution $u=x(1-x) y(1-y)$ and set $\mathbb{A}(x, y)=1$. The numerical results are reported in Table 2 .

Case 3. Let the exact solution $u=x(1-x) y(1-y)$ and set

$$
\mathbb{A}(x, y)=\left[\begin{array}{cc}
x^{2}+1 & 0 \\
0 & 1
\end{array}\right] \text {. }
$$

The numerical results are reported in Table 3.

The right-hand function $f$ in the performed numerical examples is chosen to match the given $u$ and $\mathbb{A}$. We take the parameters of the cascadic multigrid method as $\beta=3$ and $m_{L}=30$. The numerical experiments are conducted in the computer of Intel(R) Core(TM) i5-2500 CPU $3.30 \mathrm{GH}$, 
TABLE 1: Results of Case 1: $m_{L}=30, \beta=3$.

\begin{tabular}{|c|c|c|c|c|}
\hline$h$ & $n_{L}$ & $L$ & \|\|$u_{L}^{*}-u\|\|_{L}$ & Time (seconds) \\
\hline \multirow{3}{*}{$\frac{1}{64}$} & \multirow{3}{*}{49408} & 1 & $5.5032 e-02$ & 13.30 \\
\hline & & 2 & $5.5067 e-02$ & 0.92 \\
\hline & & 3 & $5.5067 e-02$ & 0.73 \\
\hline \multirow{4}{*}{$\frac{1}{128}$} & \multirow{4}{*}{197120} & 1 & $2.7503 e-02$ & 109.7200 \\
\hline & & 2 & $2.7530 e-02$ & 5.41 \\
\hline & & 3 & $2.7530 e-02$ & 4.56 \\
\hline & & 4 & $2.7530 e-02$ & 3.73 \\
\hline \multirow{5}{*}{$\frac{1}{256}$} & \multirow{5}{*}{787456} & 1 & $1.3750 e-02$ & 942.54 \\
\hline & & 2 & $1.3765 e-02$ & 30.45 \\
\hline & & 3 & $1.3766 e-02$ & 18.30 \\
\hline & & 4 & $1.3766 e-02$ & 17.10 \\
\hline & & 5 & $1.3766 e-02$ & 17.04 \\
\hline \multirow{6}{*}{$\frac{1}{512}$} & \multirow{6}{*}{3147776} & 1 & $6.8749 e-03$ & 7816.06 \\
\hline & & 2 & $6.8826 e-03$ & 180.41 \\
\hline & & 3 & $6.8830 e-03$ & 84.72 \\
\hline & & 4 & $6.8830 e-03$ & 72.63 \\
\hline & & 5 & $6.8830 e-03$ & 71.39 \\
\hline & & 6 & $6.8830 e-03$ & 70.92 \\
\hline
\end{tabular}

TABLE 2: Results of Case 2: $m_{L}=30, \beta=3$.

\begin{tabular}{|c|c|c|c|c|}
\hline$h$ & $n_{L}$ & $L$ & \|\|$u_{L}^{*}-u \|_{L}$ & Time (seconds) \\
\hline \multirow{3}{*}{$\frac{1}{64}$} & \multirow{3}{*}{49408} & 1 & $3.6360 e-03$ & 4.49 \\
\hline & & 2 & $3.6355 e-03$ & 0.75 \\
\hline & & 3 & $3.6355 e-03$ & 0.72 \\
\hline \multirow{4}{*}{$\frac{1}{128}$} & \multirow{4}{*}{197120} & 1 & $1.8181 e-03$ & 36.11 \\
\hline & & 2 & $1.8181 e-03$ & 4.10 \\
\hline & & 3 & $1.8181 e-03$ & 3.78 \\
\hline & & 4 & $1.8181 e-03$ & 3.76 \\
\hline \multirow{5}{*}{$\frac{1}{256}$} & \multirow{5}{*}{787456} & 1 & $9.0907 e-04$ & 246.85 \\
\hline & & 2 & $9.0906 e-04$ & 21.19 \\
\hline & & 3 & $9.0906 e-04$ & 17.41 \\
\hline & & 4 & $9.0906 e-04$ & 16.90 \\
\hline & & 5 & $9.0906 e-04$ & 16.87 \\
\hline \multirow{6}{*}{$\frac{1}{512}$} & \multirow{6}{*}{3147776} & 1 & $4.5454 e-04$ & 2125.11 \\
\hline & & 2 & $4.5453 e-04$ & 106.25 \\
\hline & & 3 & $4.5457 e-04$ & 75.06 \\
\hline & & 4 & $4.5457 e-04$ & 71.62 \\
\hline & & 5 & $4.5457 e-04$ & 70.23 \\
\hline & & 6 & $4.5457 e-04$ & 70.14 \\
\hline
\end{tabular}

12.0 GB, Windows 7 (x64). The time each experiment takes is also listed in the tables.

The numerical results obtained by using the conjugate gradient iteration to solve these examples directly are also provided at the rows with $L=1$. We can observe that the proposed cascadic multigrid method is optimal for both convergence rate and computation complexity, which confirms our theoretical results.

\section{Conclusion}

This paper gives error and complexity estimates of the cascadic multigrid algorithm of the weak Galerkin finite 
TABLE 3: Results of Case 3: $m_{L}=30, \beta=3$.

\begin{tabular}{|c|c|c|c|c|}
\hline$h$ & $n_{L}$ & $L$ & \|\|$u_{L}^{*}-u \|_{L}$ & Time (seconds) \\
\hline \multirow{3}{*}{$\frac{1}{64}$} & \multirow{3}{*}{49408} & 1 & $3.6360 e-03$ & 4.43 \\
\hline & & 2 & $3.6355 e-03$ & 0.94 \\
\hline & & 3 & $3.6355 e-03$ & 0.72 \\
\hline \multirow{4}{*}{$\frac{1}{128}$} & \multirow{4}{*}{197120} & 1 & $1.8181 e-03$ & 36.08 \\
\hline & & 2 & $1.8181 e-03$ & 4.10 \\
\hline & & 3 & $1.8181 e-03$ & 3.68 \\
\hline & & 4 & $1.8181 e-03$ & 3.59 \\
\hline \multirow{5}{*}{$\frac{1}{256}$} & \multirow{5}{*}{787456} & 1 & $9.0907 e-04$ & 264.49 \\
\hline & & 2 & $9.0906 e-04$ & 21.37 \\
\hline & & 3 & $9.0906 e-04$ & 17.39 \\
\hline & & 4 & $9.0906 e-04$ & 16.91 \\
\hline & & 5 & $9.0906 e-04$ & 16.92 \\
\hline \multirow{6}{*}{$\frac{1}{512}$} & \multirow{6}{*}{3147776} & 1 & $4.5454 e-04$ & 2126.06 \\
\hline & & 2 & $4.5453 e-04$ & 106.97 \\
\hline & & 3 & $4.5457 e-04$ & 74.85 \\
\hline & & 4 & $4.5457 e-04$ & 71.07 \\
\hline & & 5 & $4.5457 e-04$ & 70.65 \\
\hline & & 6 & $4.5457 e-04$ & 70.53 \\
\hline
\end{tabular}

element. The numerical solution is convergent with rate $O(h)$ and the computing time is proportional to the number of unknowns, which verify the theoretical results.

\section{Conflicts of Interest}

The authors declare that there are no conflicts of interest regarding the publication of this paper.

\section{Acknowledgments}

This work is supported by the National Science Foundation of China (Grant no. 11101311) and Applied Mathematics Chair Fund of China-German College (Grant no. 0900101021).

\section{References}

[1] J. Wang and X. Ye, "A weak Galerkin finite element method for second-order elliptic problems," Journal of Computational and Applied Mathematics, vol. 241, pp. 103-115, 2013.

[2] L. Mu, J. Wang, Y. Wang, and X. Ye, "A computational study of the weak Galerkin method for second-order elliptic equations," Numerical Algorithms, vol. 63, no. 4, pp. 753-777, 2013.

[3] L. Mu, J. Wang, and X. Ye, "Weak Galerkin finite element methods on polytopal meshes," International Journal of Numerical Analysis \& Modeling, vol. 12, no. 1, pp. 31-53, 2015.

[4] L. Mu, J. Wang, Y. Wang, and X. Ye, "A weak galerkin mixed finite element method for biharmonic equations," in Numerical Solution of Partial Differential Equations: Theory, Algorithms, and Their Applications, pp. 247-277, Springer, 2013.

[5] L. Mu, J. Wang, G. Wei, X. Ye, and S. Zhao, "Weak Galerkin methods for second order elliptic interface problems," Journal of Computational Physics, vol. 250, pp. 106-125, 2013.

[6] L. Mu, J. Wang, and X. Ye, "Weak Galerkin finite element methods for the biharmonic equation on polytopal meshes,"
Numerical Methods for Partial Differential Equations, vol. 30, no. 3, pp. 1003-1029, 2014.

[7] L. Mu, J. Wang, X. Ye, and S. Zhang, "A weak Galerkin finite element method for the Maxwell equations," Journal of Scientific Computing, vol. 65, no. 1, pp. 363-386, 2015.

[8] J. Wang and X. Ye, "A weak galerkin finite element method for the stokes equations," Advances in Computational Mathematics, vol. 275, no. 1, pp. 1-20, 2013.

[9] J. H. Bramble, Multigrid methods, Pitman Research Notes in Mathematics Series, Pitman, London, UK, 1993.

[10] F. A. Bornemann and P. Deuflhard, "Cascadic multigrid methods," in In Domain Decomposition Methods in Sciences and Engineering, Procs. 8th International Conference, pp. 205-212, Beijing, China, 1997.

[11] F. A. Bornemann and P. Deuflhard, "The cascadic multigrid method for elliptic problems," Numerische Mathematik, vol. 75, no. 2, pp. 135-152, 1996.

[12] W. B. Chen, S. B. Ling, and J. G. Huang, "The cascadic multigrid method of the mortar finite element method for second-order elliptic problems with corner singularity," Fudan University. Journal. Natural Science. Fudan Xuebao. Ziran Kexue Ban, vol. 41, no. 5, pp. 549-556, 2002.

[13] Z.-C. Shi and X. Xu, "Cascadic multigrid method for elliptic problems," East-West Journal of Numerical Mathematics, vol. 7, no. 3, pp. 199-209, 1999.

[14] P. G. Ciarlet, The Finite Element Method for Elliptic Problems, North-Holland, Amsterdam, The Netherlands, 1978. 


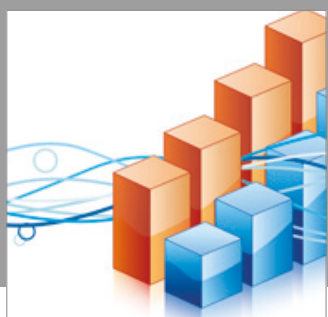

Advances in

Operations Research

vatersals

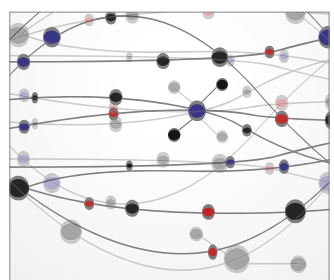

\section{The Scientific} World Journal
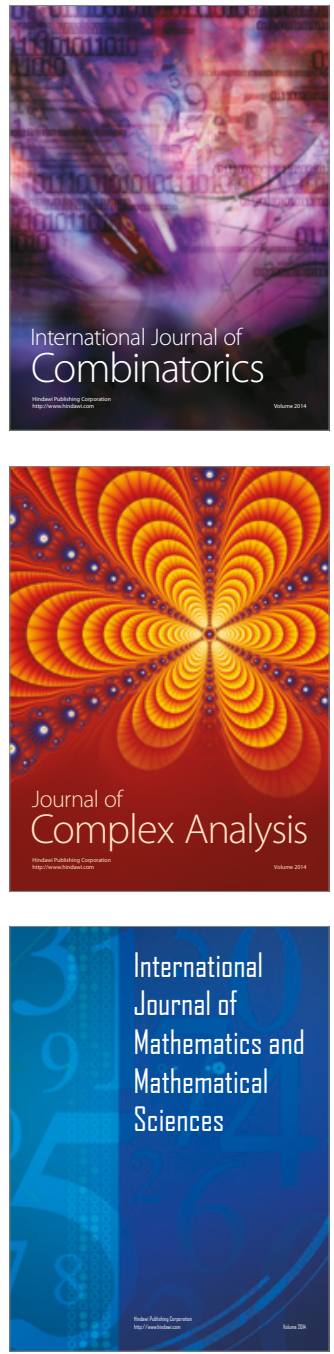
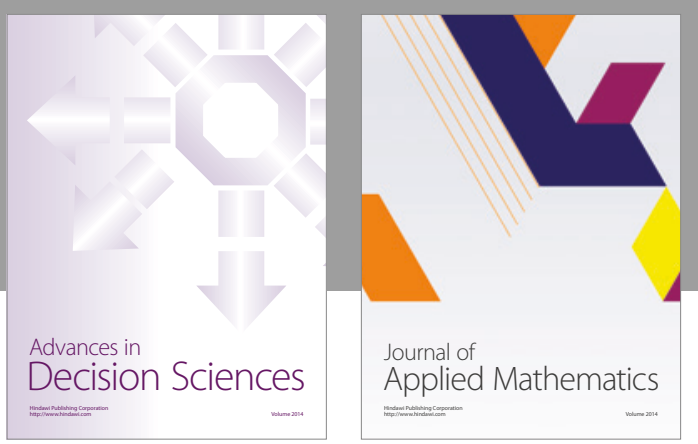

Algebra

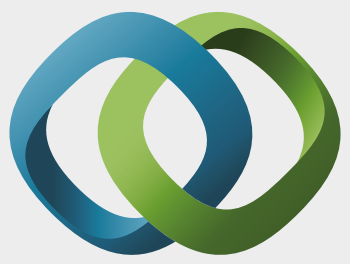

\section{Hindawi}

Submit your manuscripts at

https://www.hindawi.com
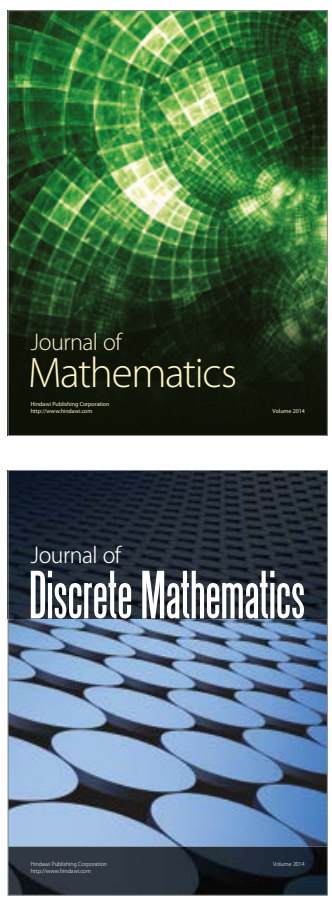

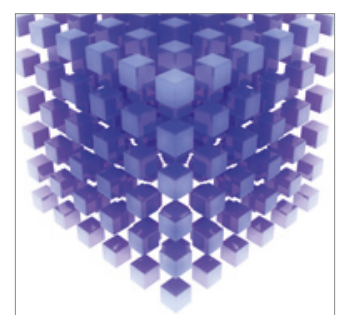

Mathematical Problems in Engineering
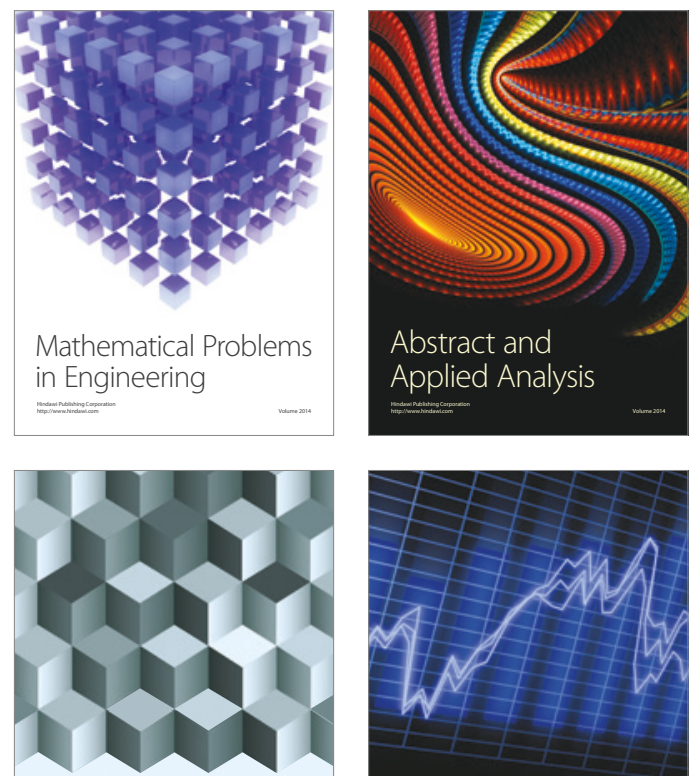

Journal of

Function Spaces

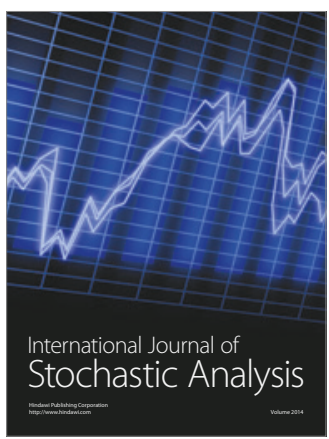

Probability and Statistics
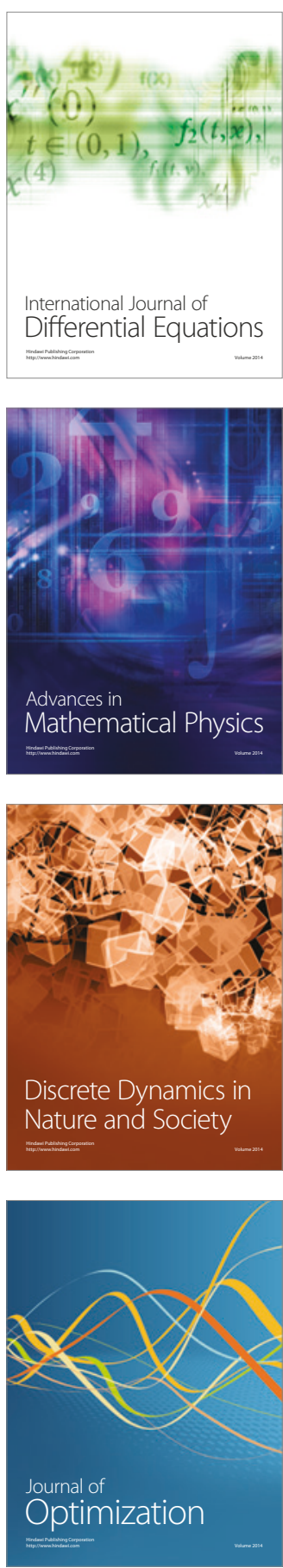